\title{
Bar dissolution and bulge formation
}

\author{
C.A. NORMAN \\ Space Telescope Science Institute and Johns Hopkins University, Baltimore, MD 21218, \\ U.S.A. \\ H. HASAN** \\ Space Telescope Science Institute, 3700 San Martin Dr., Baltimore, MD 21218, U.S.A. \\ and

\section{J.A. SELLWOOD} \\ Dept. of Physics and Astronomy, Rutgers University, Piscataway, NJ 08855-0849, \\ U.S.A.
}

We discuss the general classification of secular evolution in galaxies into terms of stellar dynamics. We present two-dimensional $N$-body simulations of a disk galaxy in which a central mass concentration is imposed after the formation of a strong bar. We show that the bar dissolves almost completely if the central mass concentration exceeds approximately $5 \%$ of the combined disk and bulge mass. This behavior can be understood in terms of previous work on single particle orbits (Hasan \& Norman 1990, Hasan et al. 1993); the sustaining orbits aligned with the bar become stochastic as the Inner Lindblad resonance moves out past the minor axis of the bar. We present arguments that bar formation and subsequent thickening and dissolution will create a bulge-like stellar distribution from the central part of the disk. We discuss the predictions of such a model including the point that barred Scs with sufficient central mass concentrations should be building bulges now. We emphasize that bulges can come from a number of different mechanisms and we discuss the current evidence at both high and low redshift.

Currently we do not know from either observational or theoretical studies whether the scenario presented by the bar-central mass dissolution model actually leads to significant late time evolution along the Hubble-sequence. Observations of galaxy morphology at high redshift with the Hubble Space Telescope will help answer this question when the high redshift distribution of galaxies along the Hubble sequence is compared to the normal low redshift distribution. Catching a galaxy in the act of bulge formation by observing a suitable barred $\mathrm{Sc}$ with a sufficiently large central mass concentration and with a Population I bulge and disk kinematics may also be very productive. For recently heated bulge components the kinematics of any young A-type stars in the bulge should be found to have cylindrical rotation.

We plan to pursue these studies by performing $3 \mathrm{D}$ N-body simulations.

\section{References}

Hasan, H. and Norman, C., 1990, ApJ 361, 69.

Iasan, H., Pfenniger, D. and Norman, C., 1993, ApJ, 409, 91.

** PRESENT ADDRESS: ASTROPHYSICS DIVISION, NASA HEADQUARTERS, WASHINGTON D.C., U.S.A. 